Meta

Journal des tradlucteurs

Translators' Journal

\title{
Droit public et traduction
}

\section{Nina Riva}

Volume 26, numéro 3, septembre 1981

URI : https://id.erudit.org/iderudit/003952ar

DOI : https://doi.org/10.7202/003952ar

Aller au sommaire du numéro

Éditeur(s)

Les Presses de l'Université de Montréal

\section{ISSN}

0026-0452 (imprimé)

1492-1421 (numérique)

Découvrir la revue

Citer cet article

Riva, N. (1981). Droit public et traduction. Meta, 26(3), 223-228.

https://doi.org/10.7202/003952ar d'utilisation que vous pouvez consulter en ligne.

https://apropos.erudit.org/fr/usagers/politique-dutilisation/ 


\section{Droit public et traduction}

Nina Riva

Dans une de ses lettres à sa sœur Laura, Eleonor Marx ${ }^{1}$ écrit :

Je t'envoie aussi ma traduction du rapport allemand. Tu verras que $j$ 'ai seulement ajouté une ou deux notes explicatives (j'avais demandé au Général $^{2}$ ce qu'il en pensait et il a été d'accord). Les Anglais ne peuvent pas savoir ce qu'est la "Gesinde Ordnung» ${ }^{3}$, bien que nous ayons quelque chose de semblable pour les ouvriers agricoles ${ }^{4}$.

La traduction dont parle Eleonor Marx est celle des différents rapports du congrès international convoqué par le Parti ouvrier belge et qui s'est ouvert à Bruxelles le 16 août 1891. La difficulté à laquelle elle est confrontée est classique en droit public, en ce sens qu'elle ne peut trouver, en anglais, un terme équivalant au terme allemand parce que l'organe parallèle est inexistant au Royaume Uni. On peut, dans un tel cas, se résigner et considérer le terme comme intraduisible parce que le concept qu'il couvre n'a pas de correspondant dans l'autre système juridique.

Il s'agirait aujourd'hui d'une attitude indéfendable et peu scientifique. Depuis la Seconde Guerre mondiale, certaines disciplines appartenant au droit public, telles que le droit politique, le droit constitutionnel et le droit administratif entre autres, ont évolué avec une rapidité déconcertante et l'interdépendance des divers systèmes n'est pas à mettre en doute. Non seulement les rapports entre gouvernants et gouvernés se sont intensifiés et ont changé, mais aussi est né, entre les différents gouvernants, ou entre les états dont ils assument le pouvoir, un nouvel ordonnancement politique, qui souvent a été à l'origine d'un nouvel ordre juridique. Par ailleurs, il existe peu d'études comparatives pour ces matières, bien moins que pour la plupart des branches du droit privé. Quiconque est confronté à ces problèmes, par nécessité professionnelle ou par intérêt scientifique, a pu se rendre compte des obstacles quelquefois difficilement franchissables qu'ils posent.

Le traducteur en fait son pain quotidien. Il rencontre ce que Georges Mounin appelle des «cultures» (ou des «civilisations») profondément différentes, qui constituent non pas autant de «visions du monde» différentes, mais autant de «mondes" réels différents ${ }^{5}$. Si l'on admet que la fonction traduisante

1. Eleonor est la fille cadette de Karl Marx. En 1979 est paru, chez Albin Michel, les Filles de Karl Marx, lettres inédites, collection Bottigelli, Michelle Perrot, Olga Meier, Michel Trebitsch.

2. Engels.

3. Législation définissant les droits et les devoirs des maîtres et des serviteurs.

4. M. Perrot, O. Meier, M. Trebitsch, op. cit., p. 281.

5. Georges Mounin (1963): les Problèmes théoriques de la traduction, Paris, Gallimard, p. 59. 
est possible - et elle l'est puisqu'elle existe - il faut trouver pour la traduction juridique, sinon la voie royale, au moins un des chemins qui doit permettre non seulement à un être $\mathrm{X}$ parlant une langue $\mathrm{A}$ de devenir $\mathrm{Y}$ parlant une langue $\mathrm{B}$, mais bien à un juriste $X$ parlant une langue $A$ spécialisée (juridique en l'occurrence) de devenir un juriste $Y$ parlant une langue $B$ spécialisée. Il s'agit bien, dans le cadre du droit public, de deux êtres appartenant à deux cultures juridicophilosophiques différentes parlant forcément deux langues spécifiques différentes et qui doivent pourtant être une seule et même personne. Parce qu'il est clair que si fonction traduisante il y a, elle doit être invisible. La perception même de la traduction, ce que Landa appelle «la conception naïve de la traduction " ${ }^{6}$ l'anéantit; l'utilisateur se sent trahi et n'y croit plus. Il préfère le texte original dont il saisit mal la langue mais dont il connaît le fond à un texte dont il comprend la langue mais dont il sait le fond douteux. Et ici on en arrive au message. Le traducteur, dont il n'est plus nécessaire de rappeler qu'il doit faire passer le message, l'esprit et non la lettre, a une tâche ardue et particulièrement délicate quand il touche à la sémantique juridique. Dans ce cas, il doit avoir une connaissance linguistique spécialisée (langue active et langue passive) ce qu'il ne peut acquérir vraiment qu'à travers l'étude des différents systèmes juridiques en langue originale.

Il doit, en outre, souvent - en droit public presque toujours - se livrer lui-même à une étude comparée des différents systèmes avant de pouvoir entamer la traduction proprement dite. C'est ainsi qu'il est indispensable, s'il a, au niveau européen, le français, l'allemand et le néerlandais comme langues de travail, qu'il sache que le «Hoge Raad der Nederlanden» n'est pas le «Conseil supérieur des Pays-Bas», mais correspond en France et en Belgique à la «Cour de cassation»; que le «Conseil Constitutionnel» français est inexistant en Belgique et aux Pays-Bas et a une partie des prérogatives qui dans ces derniers pays sont dévolues au «Conseil d'État»; que le «PS» français n'est pas seulement la traduction du «SPD» allemand, qui est à son tour plus proche de l'ancienne «SFIO»; que le mot «staatsrecht» n'est pas traduisible en français autrement que par une périphrase du style «l'état et ses institutions»; que le terme «onderminister » peut être traduit en français par «vice-ministre», «secrétaire d'état », «sous-secrétaire d'état», "ministre-adjoint», mais que malheureusement ces différents concepts correspondent à des charges ministérielles bien différentes dans l'ensemble des pays de langue française; etc. Il s'agit bien sûr de quelques exemples relevés parmi les innombrables difficultés que rencontre le traducteur de droit public, qui exigent de lui une solide connaissance juridique, un intérêt très vif pour l'évolution rapide de cette matière et une grande maîtrise de la langue de spécialité concernée. Je ne m'attarderai pas sur la différence que voient un grand nombre d'auteurs entre la langue de spécialité et la liste terminologique, même très complète ${ }^{7}$. Ce n'est pas mon propos.

Toujours est-il que le traducteur de droit public doit pouvoir parler en juriste dans ses langues de travail. Ce qui pose la question de l'existence même

6. Mariano Garcia Landa (avril 1979): Théorie générale de la traduction, version restreinte, p. 1.

7. Voir Nina Riva (1973): "Les langues de spécialités", in Dix années de linguistique théorique et appliquée, Université de l'État, Mons, p. 129-137. 
de la spécialisation. Peut-on espérer, dans le chef d'une seule personne, la connaissance approfondie de plusieurs philosophies juridico-culturelles (puisque la philosophie juridico-culturelle est à l'origine du droit public) ainsi que la connaissance, en linguiste averti, des problèmes de langue spécialisée? Ne risque-t-il pas vite d'étioler une vérité, celle du fond ou celle de la forme? Une prépondérance de l'une des deux formations, juridique ou linguistique, ne se manifestera-t-elle pas vite? Il est certain qu'un choix, mème inconscient, se fera d'après les affinités du traducteur. Cela n'a d'ailleurs aucune importance. On peut supposer qu'à ce niveau d'exigence il a pu mesurer la difficulté et souvent la gravité de sa tâche et qu'il saura apprécier ses lacunes dans l'un ou l'autre domaine. Dans les textes de très haute spécialisation le travail en binôme s'imposera quelquefois. J'entends par là des textes qui remontent jusqu'aux racines mêmes du droit étranger et que le traducteur ignore parce que sa formation double présente précisément et obligatoirement les lacunes que je viens d'évoquer.

Et la pédagogie? En d'autres mots comment faire acquérir à un aspirant traducteur juridique la maîtrise de sa future profession? Comment le familiariser avec ce droit étranger et le langage qui est le sien?

Le raisonnement juridique touche à la fois à l'objectivité et à la subjectivité des faits et du langage, ce qui le rend éminemment dépendant du lieu et du temps. L'objet du droit (et plus particulièrement du droit public) se transforme selon des variables qui souvent nous échappent. En régime démocratique, le système présidentiel a profondément changé en vingt ans; il y a aujourd'hui autant de systèmes que de pays qui l'appliquent.

Pour ces raisons et pour d'autres, inhérentes à tout enseignement, il n'existe évidemment aucune méthode scientifique idéale qui permettrait, de façon infaillible, l'acquisition d'un savoir et d'une technique aussi complexes que la traduction en droit public.

Au surplus, la formation préalable du candidat importe beaucoup. S'il s'agit d'un juriste, il faudra surtout se préoccuper de sa formation linguistique et de la technique de la traduction. $\mathrm{Si}$, au contraire, on a affaire à quelqu'un qui a une formation linguistique - ce qui est le plus souvent le cas - il faudra l'initier au dépouillement de la matière juridique par la méthode de la critique juridique comparée, en insistant sur la quantité importante de similitudes qui peuvent exister entre différents systèmes à l'intérieur du même groupe ${ }^{8}$. On ne traduit pas du droit si on ne comprend rien au droit. Le but de l'initiation est donc double: elle doit aider le traducteur à explorer sa propre culture juridique et son langage

8. On peut actuellement parler de trois grands groupes :

- le groupe occidental :

- le droit écrit (code napoléon et systèmes assimilés);

- le droit coutumier;

- le groupe marxiste :

- le système soviétique:

- le système chinois;

- le groupe religieux :

- le système sunnite;

- le système chiite;

- le système hindou

- et d'autres. 
ainsi que celle dont l'autre langue est l'expression. Comme, pas plus que pour les langues, il n'existe de système juridique isolé, la méthode comparée à partir de documents originaux s'avère, sinon la meilleure, du moins la plus rentable. Après une analyse approfondie, écrite et orale, de textes écrits par et pour des spécialistes, un juriste discutera en langue originale des divers systèmes avec les traducteurs. Le dialogue, stricto sensu, c'est-à-dire en respectant et le sujet traité et la langue y afférent, pour fructueux qu'il soit, est dur et impitoyable.

Les étudiants en langue néerlandaise de l'École d'interprètes internationaux de l'Université de Mons (Belgique) dont la langue maternelle est le français sont initiés aux systèmes juridiques français (en français), belge (en français et en néerlandais), néerlandais (en néerlandais), appartenant tous les trois au groupe occidental, droit écrit. Ils peuvent ainsi à la fois parfaire leurs connaissances linguistiques en langue maternelle et élargir leurs connaissances en langue étrangère grâce à un contenu cohérent, seule source réelle de l'apprentissage de la langue de spécialité. Cette confrontation pédagogique de deux langues et de trois systèmes juridiques peut paraître une gageure. Elle exige en effet de la part de l'enseignant la spécialisation juridique et la connaissance approfondie des deux langues. L'étudiant, en revanche, devra faire preuve d'un effort soutenu et important.

Un exemple très récent, parmi beaucoup d'autres, d'homonymie créée par la transformation profonde du droit public belge parle en faveur d'une telle méthode. Il y a peu le système politique belge $a$, dans le cadre de la réforme de l'état ${ }^{9}$, fait référence à une nouvelle forme de règlement: 1'ordonnance. Ce faisant, le législateur puise dans un vocabulaire existant du français en ce sens que le mot n'est pas neuf mais que sa signification initiale est transformée et adaptée à une nouvelle situation voulue par les gouvernants. Cet état de choses n'est évidemment pas l'apanage du langage juridique, on le rencontre aussi dans la langue courante mais il va de soi que le risque de confusion, de solécisme, de non-sens ou de contresens qu'il peut entraîner présente un danger évident en droit. Peu importe de savoir qui, dans ces cas, a le mieux respecté l'acception primaire. Le traducteur n'est ni un étymologiste ni un grammairien mais quelqu'un qui doit sur-le-champ, sans marge de sécurité aucune, produire un travail juridique faisant foi. Dans l'exemple qui nous préoccupe, cela veut dire qu'il doit savoir que l'ordonnance (belge) est une variation nationale du concept français mais dont le sens a été profondément modifié et qu'en néerlandais, le mot existe uniquement en Belgique alors qu'aux Pays-Bas, il correspond au «Algemene Maatregel van Bestuur». Cela donne le schéma suivant où s'opère un véritable chassé-croisé linguistique :

9. Il s'agit en l'occurrence de transformer l'état unitaire en un état fédéral composé de deux communautés et de deux ou trois régions. 


\begin{tabular}{ccc}
\hline FRANCE & BELGIQUE & PAYS-BAS \\
\hline Ordonnance & $\pm\left\{\begin{array}{cc}\text { Arrêté Royal } \\
\text { Koninklijk Besluit }\end{array}\right.$ & $\begin{array}{c}\text { Algemene Maatregel } \\
\text { van Bestuur }\end{array}$ \\
\hline- & Ordonnance & - \\
\hline
\end{tabular}

Le traducteur doit savoir que l'ordonnance belge n'a d'équivalent ni en France ni aux Pays-Bas, parce qu'il s'agit, dans les deux cas, d'états unitaires, l'un centralisé, l'autre décentralisé.

Il est évident qu'aucun dictionnaire aussi complet soit-il, qu'aucune liste de terminologie spécialisée même si elle est explicative et multilingue, ne pourront jamais fournir que quelques éléments de réponse qui, à leur tour, ne pourront être compris que par des gens ayant acquis une méthode scientifique d'approche des matières en question.

Comme le dit Meillet: «Tout vocabulaire exprime une civilisation. Si l'on a, dans une large mesure, une idée précise du vocabulaire français, c'est qu'on est informé sur l'histoire de la civilisation en France ${ }^{10}$. D'autre part je ne peux que souscrire totalement aux paroles de Georges Mounin quand il dit :

Supposons un traducteur français qui travaille soit sur le domaine russe, soit sur le domaine japonais, soit sur le domaine brésilien : cette expérience multiple, inorganique, inégale, hasardeuse, qu'il possède en fait, de l'ethnographie soit russe, soit japonaise, soit brésilienne, lui masque le fait théorique, qu'il faudrait énoncer ainsi, et qu'on peut énoncer ainsi : "pour traduire une langue étrangère, il faut remplir deux conditions, dont chacune est nécessaire, et dont aucune en soi n'est suffisante : étudier la langue étrangère; étudier (systématiquement) l'ethnographie de la communauté dont cette langue est l'expression. "

Nulle traduction n'est totalement adéquate si cette double condition n'est pas satisfaite.

L'ignorance de cette double condition - mieux, de ces deux conditions égales en dignité théorique - se reflète aussi dans ce fait que l'on appelle indistinctement fautes de traduction les fautes qui ressortissent à l'insuffisante connaissance de la langue étrangère et celles qui ressortissent à l'ignorance de la civilisation dont cette langue est l'expression; dans le fait même qu'on reproche au traducteur qui commet ces dernières d'ignorer la «langue qu'il traduit ${ }^{11}$.

Il est faux de croire qu'il suffit d'être linguiste, même très averti, pour être, de par là même, bon traducteur spécialisé. Il est faux de croire que de bons dictionnaires spécialisés sont une panacée; ils ne sont jamais, et ce dans le meilleur des cas, qu'une codification de termes qui recouvrent une situation non vécue par le traducteur.

10. Georges Mounin, op. cit., p. 235.

11. Georges Mounin, op. cit., p. 236. 
Le choix plus ou moins hasardeux opéré par le traducteur peut, selon la matière à traduire, créer des situations ridicules ou fausses; en droit, elles risquent d'être dangereuses.

En guise de conclusion, on peut dire qu'il est vrai que la connaissance des langues n'est qu'une béquille pour le traducteur. Il a besoin de deux autres voies pour accéder à la signification réelle des choses : 1'arrière-plan socio-culturel et la technique de la traduction.

En droit, l'étude de l'arrière-plan socio-culturel est une spécialité en soi. 